\title{
Caracterização hidráulica do microaspersor Rondo, da Plastro'
}

\author{
Roberto S.F. de Holanda Filho ${ }^{2}$, Francisco de Q. Porto Filho ${ }^{3}$, Neyton de O. Miranda ${ }^{3}$ \& José F. de Medeiros ${ }^{4}$ \\ 1 Monografia apresentada pelo primeiro autor à ESAM, para obtenção do título de Engenheiro Agrônomo \\ 2 UFC, Campus do Pici - Bloco 804 Pici, CEP 60455-600, Fortaleza, CE. E-mail: rsfhf@bol.com.br (Foto) \\ ${ }^{3}$ ENA-ESAM. C.P. 137, CEP 59625-900, Mossoró, RN. E-mail: engeagri@esam.br \\ ${ }^{4}$ ENA-ESAM. E-mail: jfmedeir@esam.br
}

Protocolo 057 - 29/05/2000

\begin{abstract}
Resumo: Com o objetivo de se determinar as características hidráulicas do microaspersor Rondo $50 \mathrm{~L} \mathrm{~h}^{-1}$, de bocal azul, fabricado pela Plastro, conduziram-se testes no Laboratório de Hidráulica da Escola Superior de Agricultura de Mossoró, determinando-se a equação característica vazão - pressão, o coeficiente de variação de fabricação (CVf), o perfil de distribuição, o diagrama pluviométrico e a uniformidade de distribuição de água pelo microaspersor. A equação característica obtida foi $q=3,4312 \mathrm{H}^{0,516}$, caracterizando fluxo turbulento; o CVf médio foi considerado excelente e o microaspersor apresentou um raio de alcance igual a 3,3 m. O espaçamento com melhor uniformidade de distribuição e superposição, foi o de 1,2 x 1,2 m, embora para espaçamentos de até 3,6 x 3,6 m, a uniformidade de distribuição tenha sido superior a $80 \%$.
\end{abstract}

Palavras-chave: microirrigação, uniformidade de distribuição, equação característica

\section{Hydraulic characterization of the Plastro Rondo microsprinkler}

\begin{abstract}
In order to assess the hydraulic characteristics of the microsprinkler Rondo $50 \mathrm{~L} \mathrm{~h}^{-1}$, with blue nozzle, manufactured by Plastro, tests were carried out in the Hydraulic Laboratory of the Escola Superior de Agricultura de Mossoró (ESAM). Estimates were made for discharge pressure head equation, manufacturer's coefficient of variation, profile of distribution, uniformity of water application and water application uniformity with overlap. The manufacture's coefficient of variation is classified as excellent and the characteristic equation is $\mathrm{q}=3.4312 \mathrm{H}^{0.516}$, characterizing a turbulent flow. The microsplinker has a throw radius of a $3.3 \mathrm{~m}$. The best emitter spacing with overlap found was $1.2 \times 1.2 \mathrm{~m}$, although for spacing up to $3.6 \times 3.6 \mathrm{~m}$ the application uniformity was above $80 \%$.
\end{abstract}

Key words: microirrigation, application uniformity, discharge equation

\section{INTRODUÇÃO}

A irrigação localizada caracteriza-se pela aplicação de água diretamente sobre a região radicular, com pequenas vazões e alta freqüência, de modo a manter um conteúdo adequado de umidade no volume de solo molhado (Lopez et al., 1992). Na irrigação localizada, a água é filtrada, conduzida ao solo e, ocasionalmente, fertilizada, através de peças especiais denominadas emissores ou aplicadores, sob forma de gotas, filetes de água, pequenos jatos ou borrifos (Lima \& Azevedo, 1991).

Na microaspersão, a água é aspergida em pequenos círculos ou setores, junto ao pé da planta, cuja condução é feita por rede de tubos que operam a baixas pressões $(100$ a $300 \mathrm{kPa})$ porém superiores às do gotejamento. As vazões, que variam de 20 a $120 \mathrm{~L} \mathrm{~h}^{-1}$, e as áreas molhadas por cada microaspersor são superiores às dos gotejadores (Telles, 1986). Deve-se dar preferência à microaspersão em vez de gotejo em solos muito permeáveis, sob pena de não se obter o bulbo úmido mínimo necessário para as culturas (Pizarro, 1990). Sendo os emissores um dos componentes de maior importância, tanto no dimensionamento como no manejo dos sistemas de irrigação localizada, é de fundamental importância que os projetistas conheçam suas características (Bernardo, 1987).

A vazão do emissor, de acordo com Olitta (1987), pode ser representada pela equação $\mathrm{q}=\mathrm{kH}^{\mathrm{x}}$, em que: $\mathrm{q}$ é a vazão $\mathrm{em} \mathrm{L} \mathrm{h}^{-1}$, $\mathrm{H}$ é a pressão de operação em $\mathrm{kPa}, \mathrm{k}$ é o coeficiente de proporcionalidade (adimensional) e x é o expoente de descarga. Segundo Pizarro (1990) um emissor perfeito teria o expoente $\mathrm{x}=0$ (autocompensante), os de regime laminar $\mathrm{x}=1 \mathrm{e}$ nos de regime turbulento $\mathrm{x}<1$; já Keller \& Karmeli (1974) consideram de regime laminar os emissores com expoente $\mathrm{x}=1$ e de regime turbulento com $\mathrm{x}=0,5$.

Qualquer variação na vazão dos emissores na área do projeto poderá afetar a lâmina aplicada às plantas. Abreu et al. (1987) afirmam que o desempenho hidráulico de um emissor é determinado, dentre outros fatores, pelo coeficiente de variação 
de fabricação, que é uma medida de variação de fluxo causada pela variação no processo de fabricação. O coeficiente de variação de fabricação pode afetar mesmo um projeto corretamente dimensionado (Olitta, 1986).

Por mais precisos que sejam os processos de fabricação, os emissores apresentam diferenças milimétricas, cujas conseqüências se refletem nos valores do coeficiente de descarga, ' $k$ ', e no expoente de descarga, ' $x$ ' (Azevedo, 1986). De acordo com Dantas Neto et al. (1997), pequenas diferenças entre dois emissores aparentemente idênticos podem causar variações significativas na vazão do sistema. Por mais esmerados que sejam os processos de fabricação, sempre ocorrem variações e, como a área de escoamento dos emissores é de milímetros quadrados, qualquer variação poderá afetar as vazões dos emissores a uma mesma pressão (Costa, 1994). Nakayama \& Bucks (1981) citam as principais causas de variação de fabricação, como sendo: variação na pressão e temperatura da água; heterogeneidade do material utilizado e o projeto do próprio emissor.

O coeficiente de variação de fabricação (CVf) é uma medida estatística que avalia a variação do processo de fabricação dos emissores; tem sido utilizado, também, para avaliar a variação de fluxo do emissor ao longo de uma linha lateral de irrigação localizada. Apesar de ser impossível a fabricação de um grupo de emissores com o mesmo coeficiente de descarga, a variação resultante do processo de fabricação normalmente tende a distribuir-se em torno de um valor médio (Keller \& Karmeli, 1974; Bralts et al., 1981). De acordo com Solomon (1979) o coeficiente de variação de fabricação é o melhor parâmetro para a avaliação das diferenças individuais entre os emissores; também, é uma informação que representa bem o projeto do emissor, materiais usados na sua construção e cuidados aplicados na sua fabricação. A variação da vazão do emissor, resultado da variação de fabricação, segue a distribuição normal de Gauss; deste modo, o CVf pode ser definido pela razão entre o desvio-padrão da vazão do emissor e sua vazão média, conforme a equação:

$$
\mathrm{CVf}=100 \frac{\sqrt{\left(\mathrm{q}_{1}^{2}+\mathrm{q}_{2}^{2}+\ldots+\mathrm{q}_{\mathrm{n}}^{2}-\mathrm{nq}_{\mathrm{m}}^{2}\right) /(\mathrm{n}-1)}}{\mathrm{q}_{\mathrm{m}}}
$$

em que:

CVf - coeficiente de variação de fabricação (\%)

$\mathrm{q}_{1}, \mathrm{q}_{2}, \ldots \mathrm{q}_{\mathrm{n}}$ - vazão de cada emissor em $\mathrm{L} \mathrm{h}^{-1}$

$q_{m}$ - vazão média dos emissores em $\mathrm{L} \mathrm{h}^{-1}$

n - número de emissores do lote de amostragem.

A quantidade d'água aplicada e sua uniformidade de distribuição pelos emissores são informações importantes para o dimensionamento e manejo racional de um sistema de irrigação localizada (Nascimento et al., 1999). A utilização de emissores com boas características hidráulicas em projetos hidraulicamente bem dimensionados, proporcionarão a obtenção de bom coeficiente de uniformidade de distribuição de água e, conseqüentemente, alta eficiência de irrigação (Solomon, 1979). A distribuição de água de um sistema de irrigação localizada depende dos seguintes fatores: da variação da pressão na rede hidráulica, do coeficiente de variação de fabricação, do número de emissores por planta, da variação das características do emissor com o uso (entupimento e/ou envelhecimento), do efeito do vento e da variação de fabricação dos reguladores de pressão, quando utilizados (Sampaio et al., 1996). A uniformidade de distribuição pode ser utilizada tanto para fins de dimensionamento do sistema como para sua avaliação no campo. No caso de dimensionamento, ao contrário da avaliação de campo, a uniformidade é uma condição imposta; quanto mais alta a uniformidade, mais oneroso fica o sistema, já que são necessários maior diâmetro de tubulação, laterais mais curtas e maior investimento em reguladores de pressão (Pizarro, 1990).

No sistema de irrigação localizada, a uniformidade de aplicação d'água ao longo da linha lateral está intimamente relacionada com a variação de vazão dos emissores, a qual é uma conseqüência das perdas de energia pelo atrito e pelas inserções dos emissores, com o ganho ou perda de energia devido à topografia da superfície do solo, e com a qualidade da matéria-prima e dos processos de fabricação dos emissores (Keller \& Karmeli, 1974). Sampaio et al. (1996) trabalhando com microaspersores, concluem que os emissores equipados com dispositivo giratório possuem melhor distribuição, enquanto Dantas Neto et al. (1997) o que caracteriza e determina o funcionamento do microaspersor sobre uma superfície irrigada, é o ensaio de distribuição pluviométrica. As curvas pluviométricas indicam a precipitação horária sobre o terreno, enquanto as isoietas delimitam a área molhada, por diferentes precipitações.

O parâmetro utilizado para determinar a uniformidade de distribuição é o coeficiente de uniformidade de Christiansen (1942) expresso pela seguinte equação:

$$
\text { CUC }=100\left[1,0-\left(\sum_{i=1}^{n}\left|x_{i}-\bar{x}\right| / n \bar{x}\right)\right]
$$

em que:

CUC - coeficiente de uniformidade de Christiansen (\%)

n - número de observações

$\mathrm{x}_{\mathrm{i}} \quad$ - lâmina coletada em cada observação i (mm)

$\overline{\mathrm{x}} \quad$ - lâmina média coletada $(\mathrm{mm})$.

Nakayama \& Bucks (1986) afirmam que este coeficiente pode ser usado também para irrigação localizada.

Como em sistemas por aspersão, os aspersores podem receber disposições geométricas como quadrados, retângulos e triângulos, espaçados dentro de certas distâncias, de maneira a permitir uma perfeita distribuição de água sobre o terreno (Ollita, 1987) o mesmo deve ocorrer para microaspersores. Para isto e segundo Bernardo (1987) tem-se que considerar o tipo de arranjamento no campo (retangular, quadrado ou triangular), simular as diversas combinações de espaçamento entre eles, ao longo da linha lateral e entre linhas laterais, fazer a superposição para cada combinação de espaçamento e calcular a uniformidade com os totais superpostos em cada pluviômetro.

O objetivo deste trabalho foi avaliar as características hidráulicas do microaspersor Rondo $50 \mathrm{~L} \mathrm{~h}^{-1}$, fabricado pela Plastro, quando submetido a diversas pressões situadas dentro da faixa recomendada pelo fabricante, determinando como se 
comportam a vazão, o coeficiente de variação de fabricação (CVf), a distribuição de água e o coeficiente de uniformidade utilizando superposição para diversos espaçamentos.

\section{MATERIAL E MÉTODOS}

O presente trabalho foi realizado no Laboratório de Hidráulica do Departamento de Engenharia Agrícola da Escola Superior de Agricultura de Mossoró, utilizando-se o microaspersor Rondo $50 \mathrm{~L} \mathrm{~h}^{-1}$, não regulável, apresentando bocal azul, fabricado pela Plastro, que com a finalidade de determinar a equação característica vazão-pressão; a uniformidade de vazão (coeficiente de variação de fabricação CVf); o perfil de distribuição de água; a uniformidade de distribuição de água pelo microaspersor e o coeficiente de uniformidade de distribuição de água, com superposição, realizou-se dois ensaios.

No primeiro ensaio, seguindo as recomendações da ABNT (1986), coletou-se, em revendedores, uma amostra aleatória de 20 microaspersores, os quais tiveram suas vazões determinadas quando submetidos às pressões de 50,100,150, 200, $250 \mathrm{e}$ $300 \mathrm{kPa}$, com três repetições para cada uma. Utilizou-se uma bancada (Figura 1) cujo abastecimento de água para o sistema ocorreu por bombeamento de um reservatório com capacidade de 200 L. A água após passar por um filtro de tela de 155 mesh era conduzida por uma tubulação de polietileno, contendo saídas para os microaspersores, que percorria toda a bancada e tinha conectado no seu final um manômetro eletrônico, modelo Hitronic, com precisão de $\pm 0,5 \% \mathrm{FE}$, seguido de um registro de esfera para controle da pressão.

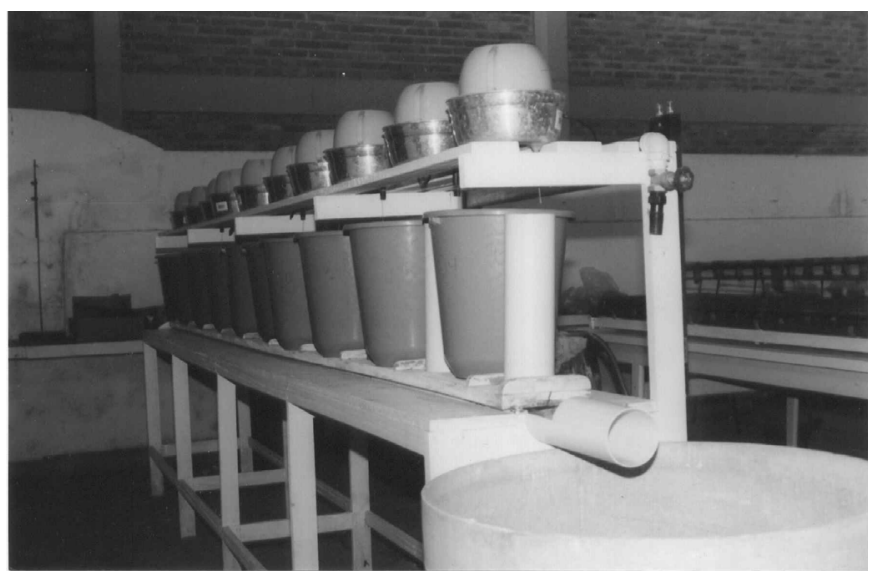

Figura 1. Foto da bancada de testes para os ensaios da curva vazão - pressão e CVf

$\mathrm{Na}$ bancada eram colocados em funcionamento, simultaneamente, 10 microaspersores e sobre cada um deles havia uma campânula de plástico que interceptava o jato d'água e direcionava o volume aplicado para baldes plásticos com capacidade de 20 L, conforme mostrado na Figura 1. O tempo de coleta variou com a capacidade dos baldes que conforme a ABNT (1986) não deve ser inferior a $15 \mathrm{~L}$ e com a pressão utilizada. Após a coleta, os baldes foram pesados em uma balança analógica, com capacidade de $20 \mathrm{~kg}$ e resolução de $0,02 \mathrm{~kg}$ e o peso foi transformado em volume, ao dividi-lo pela densidade da água determinada antes de cada repetição.
Com os valores médios de vazão, das três repetições das 20 unidades de microaspersores testados neste ensaio, determinou-se, através de análise de regressão, a curva característica vazão - pressão. E, com os mesmos dados, determinou-se o coeficiente de variação de fabricação (CVf) utilizando-se a Eq. (1). A uniformidade de vazão estabelecida foi a média dos coeficientes de variação de vazão obtidos para cada pressão adotada.

Para a realização da análise de variância visando-se verificar o efeito da pressão no coeficiente de variação de fabricação e na vazão do microaspersor, utilizaram-se os valores dos coeficientes de variação de fabricação calculados para cada repetição na referida pressão. Utilizou-se, para análise desses dados, o programa computacional SAEG (1993).

No segundo ensaio foram realizados testes com ausência de vento, com o objetivo de se determinar o perfil de distribuição da água e o raio de alcance do jato do microaspersor. Durante os testes, a umidade relativa e a temperatura, medidas na estação meteorológica Jerônimo Rosado da ESAM variaram, respectivamente, de 52,8 a $58,0 \%$ e 29,2 a $33,6^{\circ} \mathrm{C}$, enquanto o emissor escolhido para determinação do perfil de distribuição entre os 20 utilizados nos testes de vazão-pressão, foi aquele cuja vazão se aproximou mais da média da vazão para a pressão de $200 \mathrm{kPa}$. Foram realizadas três repetições. A precipitação foi coletada durante uma hora em coletores do tipo C1, da FABRIMAR, com altura de $7,87 \mathrm{~cm}$ e diâmetro de $7,98 \mathrm{~cm}$, dispostos em duas linhas ortogonais, que se cruzavam no microaspersor, o qual ficou $25 \mathrm{~cm}$ acima da seção de entrada dos coletores. A medição se deu em provetas de $14 \mathrm{~mm}$, graduadas em $0,5 \mathrm{~mm}$, adaptadas para os coletores. Nos primeiros $1,2 \mathrm{~m}$ do microaspersor os coletores estavam dispostos a $0,15 \mathrm{~m}$, e $0,3 \mathrm{~m}$ a partir deste ponto, cujos valores de precipitação para cada ponto equidistante do microaspersor foram obtidos através da média aritmética das três repetições e com os dados de precipitação do ensaio para determinação do perfil de distribuição, considerando-se os coletores dispostos de 0,6 x 0,6 m, segundo a ABNT (1986) obteve-se uma superfície de distribuição de água em milímetros por hora, pela média aritmética de três repetições. Com esses valores de precipitação, procedeu-se a representação tridimensional da distribuição de água na superfície irrigada e determinou-se o seu coeficiente de uniformidade de distribuição, utilizando-se a Eq. (2). Também, com esses mesmos dados simularam-se, de forma idêntica à aspersão, os microaspersores funcionando sobrepostos com diversos espaçamentos $(1,2 \times 1,2 ; 1,2 \times 1,8 ; 1,8 \times 1,8 ; 1,8 \times 2,4$; $2,4 \times 2,4 ; 2,4 \times 3,0 ; 3,0 \times 3,0 ; 3,0 \times 3,6 ; 3,6 \times 3,6 \mathrm{~m})$; e para esses espaçamentos, em quadrículas de $0,6 \times 0,6 \mathrm{~m}$, determinou-se o coeficiente de uniformidade, utilizando-se a Eq. (2).

\section{RESULTADOS E DISCUSSÃO}

Na Tabela 1 estão os dados de vazão média, desvio-padrão e coeficiente de variação de fabricação para as pressões preestabelecidas nos ensaios. Verifica-se que o microaspersor Rondo $50 \mathrm{~L} \mathrm{~h}^{-1}$ apresentou CVf variando entre 2,5 e 3,1\%, com valor médio de $2,8 \%$. Solomon (1979) classifica os emissores, quanto à uniformidade, da seguinte maneira: nos de CVf até 0,03 , a uniformidade é excelente; de 0,04 a 0,07 , é média; de 0,08 a $0,1 \%$, é marginal; de 0,11 a $0,14 \%$ é pobre e, acima de $0,15 \%$, 
é inaceitável, enquanto para a ABNT (1986) os de CVf inferior a $10 \%$ têm uniformidade boa; de 10 a $20 \%$ é média; de 20 a $30 \%$ é marginal e superior a $30 \%$ é inaceitável. Portanto, conforme resultados de coeficiente obtido, o microaspersor Rondo $50 \mathrm{~L} \mathrm{~h}^{-1}$ é classificado como bom pela ABNT (1986) e excelente, segundo Solomon (1979), demonstrando pequena variação na vazão devido ao processo de fabricação.

Tabela 1. Valores de vazão média, desvio-padrão, coeficiente de variação do fabricante e classificação nas pressões préestabelecidas para o microaspersor Rondo $50 \mathrm{~L} \mathrm{~h}^{-1}$

\begin{tabular}{cccccc}
\hline \multirow{2}{*}{$\begin{array}{c}\text { Pressão } \\
(\mathrm{kPa})\end{array}$} & $\begin{array}{c}\text { Vazão } \\
\text { Méd. }\left(\mathrm{Lh}^{-1}\right)\end{array}$ & $\begin{array}{c}\text { Desvio } \\
\text { Padrão }\end{array}$ & $\begin{array}{c}\text { CVf } \\
(\%)\end{array}$ & $\begin{array}{c}\text { ABNT } \\
(1986)\end{array}$ & $\begin{array}{c}\text { Solomon } \\
(1979)\end{array}$ \\
\hline 50 & 26,29 & 0,66 & 2,5 & Bom & Excelente \\
100 & 36,75 & 0,95 & 2,5 & Bom & Excelente \\
150 & 45,57 & 1,23 & 2,7 & Bom & Excelente \\
200 & 52,99 & 1,66 & 3,1 & Bom & Excelente \\
253 & 59,31 & 1,78 & 3,0 & Bom & Excelente \\
300 & 65,62 & 1,98 & 3,0 & Bom & Excelente \\
\hline
\end{tabular}

O resultado da análise de variância para avaliação do efeito da pressão no coeficiente de variação de fabricação, mostrou efeito linear da pressão sobre o CVf. Observa-se tendência de aumento do CVf a medida em que a pressão aumentou, embora ocorra variação apenas entre 2,5 e 3,1\% (Figura 2).

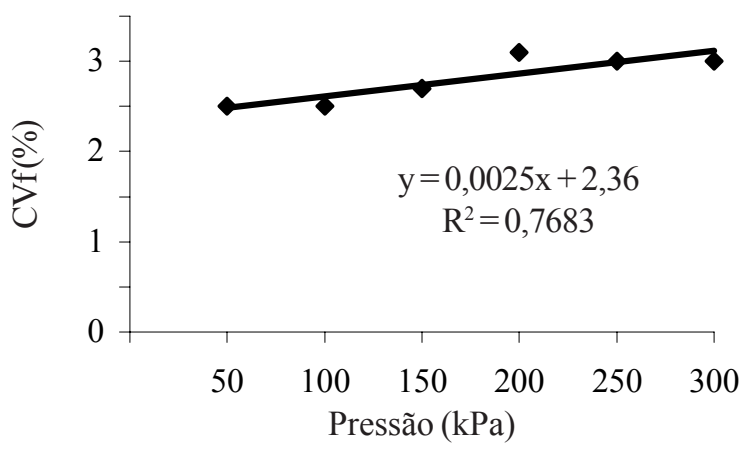

Figura 2. Valores médios dos coeficientes de variação de fabricação em função das diferentes pressões aplicadas ao microaspersor Rondo $50 \mathrm{~L} \mathrm{~h}^{-1}$

A partir dos dados de vazão coletados em laboratório e sua respectiva pressão determinou-se, por regressão, a equação característica do microaspersor, sendo:

$$
\mathrm{q}=3,4312 \mathrm{H}^{0,516} \quad\left(\mathrm{R}^{2}=0,996\right)
$$

em que:

$$
\begin{array}{ll}
\mathrm{q} & \text { - vazão do emissor }\left(\mathrm{L} \mathrm{h}^{-1}\right) \\
\mathrm{H} & \text { - pressão na entrada do emissor }(\mathrm{kPa}) .
\end{array}
$$

O coeficiente de determinação foi de 0,996 , demonstrando ótimo ajuste dos dados observados (Figura 3).

O expoente ' $x$ ' da equação característica foi igual a $0,516, \mathrm{o}$ que caracteriza, segundo Keller \& Karmelli (1974) um emissor de fluxo turbulento. $\mathrm{O}$ teste $\mathrm{F}$ mostrou-se significativo a nível de 0,01 de probabilidade.

Na Figura 4 está representado o perfil de distribuição, na qual se observa valores baixos próximo do microaspersor

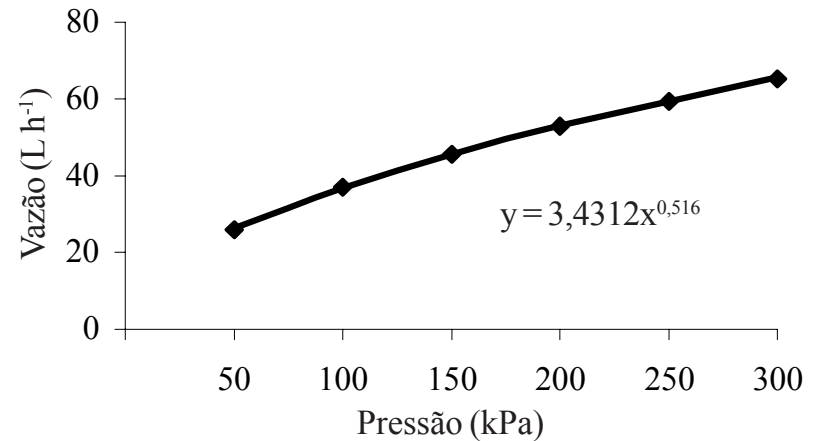

Figura 3. Curva característica vazão - pressão para o microaspersor Rondo $50 \mathrm{~L} \mathrm{~h}^{-1}$

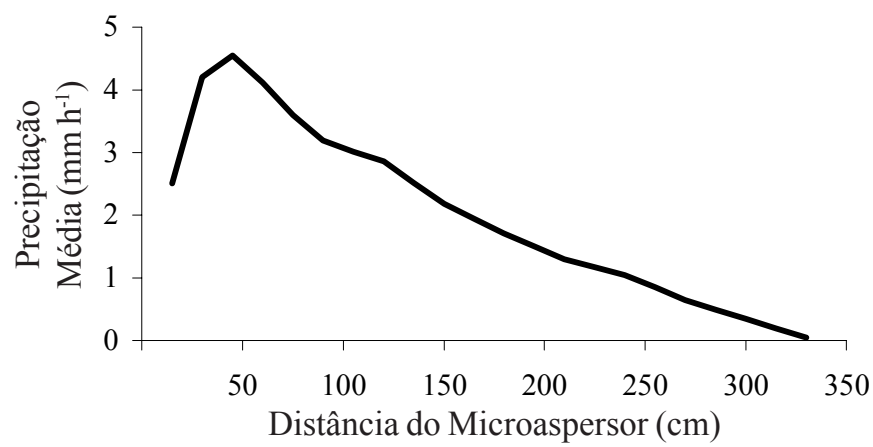

Figura 4. Perfil de distribuição de água do microaspersor Rondo $50 \mathrm{~L} \mathrm{~h}^{-1}$, operando em condições de laboratório sob pressão de $200 \mathrm{kPa}$

seguido de maiores valores da precipitação ainda próximo ao microaspersor (até $50 \mathrm{~cm}$ ), diminuindo a medida em que se distanciava deste. Costa (1994) analisando microaspersores em laboratório, observou maior concentração da precipitação junto ao microaspersor; já Nascimento et al. (1999) observaram que, próximo ao microaspersor Rain-Bird QN-14, ocorria uma zona de baixa precipitação.

O microaspersor estudado apresentou um perfil de distribuição em que o raio de alcance médio, para pressão de $200 \mathrm{kPa}$, chegou aos 3,3 m diferindo do valor anunciado pelo fabricante de $3,75 \mathrm{~m}$. A Figura 5 mostra o perfil tridimensional da distribuição de água do microaspersor, em conformidade com a Figura 4. Para este perfil de distribuição obteve-se um coeficiente de uniformidade de distribuição da ordem de 11,2\%, para um grid de 6,6 x 6,6 m. Nascimento et al. (1999) encontraram, em seus estudos com o microaspersor Rain-Bird QN-14, um coeficiente de 15,43\%, em condições semelhantes.

Para as superposições propostas, verificou-se que a melhor uniformidade ocorreu no espaçamento 1,2 x 1,2 m (Tabela 2); observa-se redução no CUC com o aumento do espaçamento dos microaspersores, embora espaçamentos de 3,6 x 3,6 m, ainda proporcionem CUC superiores a $80 \%$, sendo o valor

Tabela 2. Coeficiente de uniformidade de distribuição nos espaçamentos propostos, para o microaspersor Rondo $50 \mathrm{~L} \mathrm{~h}^{-1}$

\begin{tabular}{cccccc}
$\begin{array}{c}\text { Espaçamento } \\
(\mathrm{m})\end{array}$ & $\begin{array}{c}\text { CUC } \\
(\%)\end{array}$ & $\begin{array}{c}\text { Espaçamento } \\
(\mathrm{m})\end{array}$ & $\begin{array}{c}\text { CUC } \\
(\%)\end{array}$ & $\begin{array}{c}\text { Espaçamento } \\
(\mathrm{m})\end{array}$ & $\begin{array}{c}\text { CUC } \\
(\%)\end{array}$ \\
\hline $1,2 \times 1,2$ & 96,6 & $1,8 \times 2,4$ & 92,1 & $3,0 \times 3,0$ & 90,1 \\
$1,2 \times 1,8$ & 92,4 & $2,4 \times 2,4$ & 91,9 & $3,0 \times 3,6$ & 87,5 \\
$1,8 \times 1,8$ & 93,6 & $2,4 \times 3,0$ & 91,1 & $3,6 \times 3,6$ & 85,2 \\
\hline
\end{tabular}




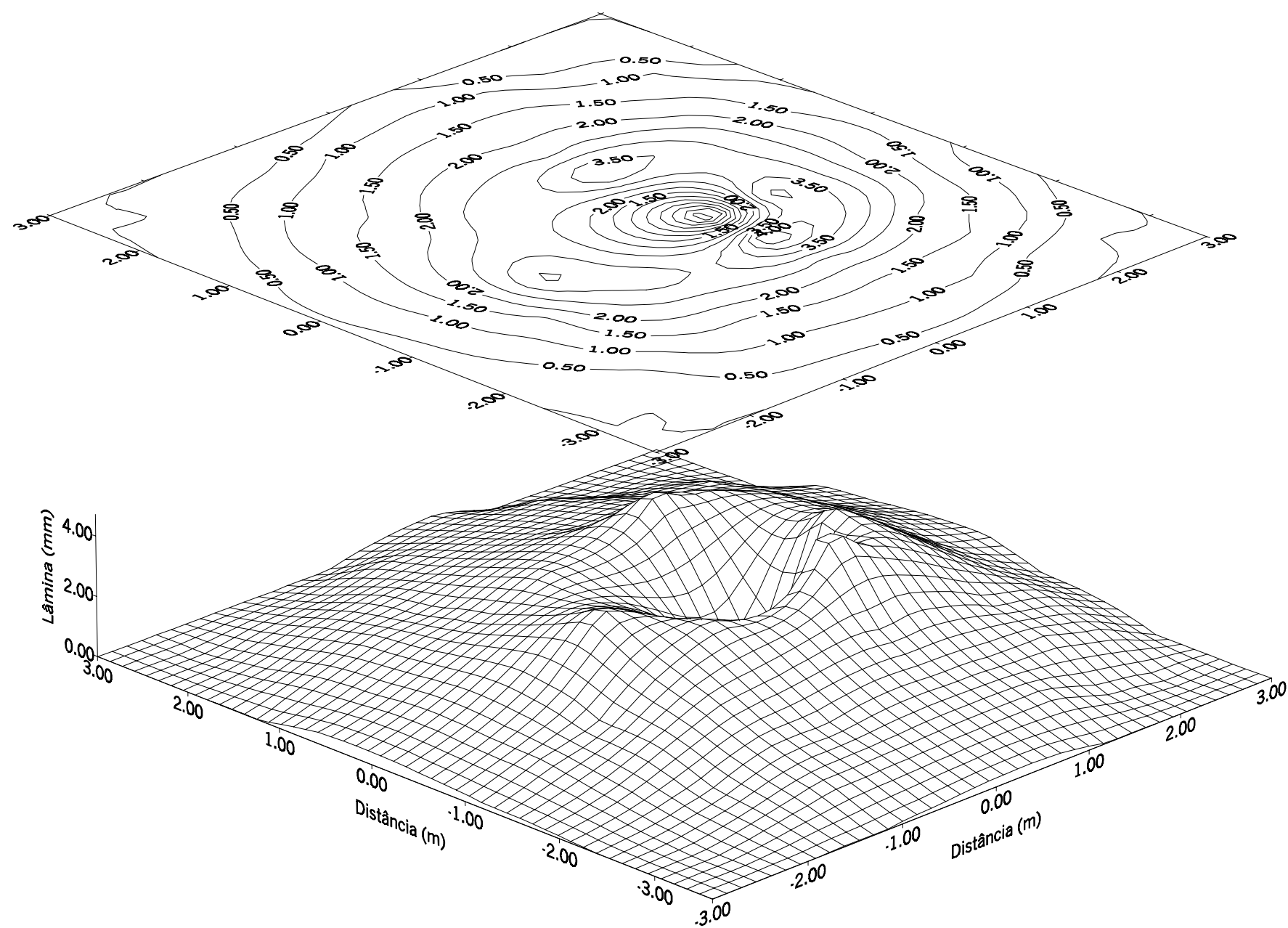

Figura 5. Diagrama pluviométrico do microaspersor Rondo $50 \mathrm{~L} \mathrm{~h}^{-1}$, operando sob uma pressão de $200 \mathrm{kPa}$, em ausência de vento

mínimo recomendável para um sistema de irrigação, segundo Bernardo (1987). Para Gomes (1999) nos sistemas de aspersão convencional os coeficientes de uniformidade variam entre 80 e $95 \%$, admitindo valores abaixo de $80 \%$ se a área a irrigar recebe chuvas durante o ciclo, se a planta possui raízes longas, ou se a economia obtida no custo da instalação (maiores espaçamentos entre aspersores e linhas) compensa a redução do rendimento da cultura irrigada.

\section{CONCLUSÕES}

1. O microaspersor Rondo $50 \mathrm{~L} \mathrm{~h}^{-1}$ apresenta coeficiente de variação de fabricação excelente.

2. O regime de escoamento do emissor é turbulento.

3. O raio de alcance do microaspersor foi de $3,3 \mathrm{~m}$, inferior ao indicado pelo fabricante.

4. O espaçamento de $1,2 \times 1,2 \mathrm{~m}$ apresentou o melhor coeficiente de distribuição de água, embora se possa recomendar espaçamentos de até 3,6 x 3,6 m.

\section{LITERATURA CITADA}

ABNT - Associação Brasileira de Normas Técnicas. Requisitos mínimos para elaboração de projeto de sistema de irrigação localizada. São Paulo, ABNT, 1986, 8p. PNBR 12:02.08 - 022.
Abreu, J.M.H.; Lopez, J.R.; Regalado, A.P.; Hernandez, J.F.G. El riego localizado. Madrid: Instituto Nacional de Investigaciones Agrárias, 1987.317p.

Azevedo, H.M. Irrigação localizada. Informe Agropecuário, Belo Horizonte, n.139, p.40-53, 1986.

Bernado, S. Manual de irrigação. 4 ed. Viçosa: UFV, 1987. 488p.

Bralts, V.F.; Wu, I.P.; Gitlin, H.M. Manufacturing variation and drip irrigation uniformity. Transactions of the ASAE, St. Joseph, v.24, n.1, p.113-119, Jan/Feb., 1981.

Christiansen, J.E. Irrigation by sprinkling. Berkeley: University of California, 1942. 124p.

Costa, M. da C. Caracterização hidráulica de dois modelos de microaspersores associados a três reguladores de fluxo e um mecanismo de pulso. Piracicaba: ESALQ, 1994. 109p. Dissertação Mestrado.

Dantas Neto, J.; Medeiros, M.G.A. de; Azevedo, C.A.V. de; Azevedo, H.M. de. Performance hidráulica e perfil de distribuição de água do microaspersor NAAN 7110, sob diferentes condições de vento. Revista Brasileira de Engenharia Agrícola e Ambiental, Campina Grande, v.1, p.57-61, 1997.

Gomes, H.P. Hidráulica dos sistemas pressurizados: Aspersão e gotejamento. 3 ed. Campina Grande: Universidade Federal da Paraíba, 1999. 412p.

Keller, J.; Karmeli, D. Trickle irrigation design parameters. Transactions of the ASAE, St. Joseph, v.17, n.4, p.878-880, 1974. 
Lima, V.L.A.; Azevedo, H.M. Caracterização hidráulica do emissor microaspersor/microtubo. In: Congresso Nacional de Irrigação e Drenagem, 9, 1991, Natal. Anais... Natal: Associação Brasileira de Irrigação e Drenagem, 1991. p.3-18.

Lopez, J.R.; Abreu, J.M.H.; Regalado, A.P.; Hernandez, J.F.G. Riego localizado. Madrid: Mundi-Prensa, 1992. 405p.

Nakayama, F.S.; Bucks, D.A. Emitter clogging effects on trickle irrigation uniformity. Transactions of the ASAE, St. Joseph, v.1, n.24, p.77-80, 1981.

Nakayama, F.S.; Bucks, D.A. Trickle irrigation for crop production: Design, operation and management. Amsterdam, Elsevier. 1986. 384p.

Nascimento, T.; Soares, J.M.; Azevedo, C. A.V. de. Caracterização hidráulica do microaspersor Rain-Bird QN-14. Revista Brasileira de Engenharia Agrícola e Ambiental, Campina Grande, v.3, n.1, p.30-33. 1999.

Olitta, A.F.L. Projeto de irrigação localizada. In: Curso de elaboração de projetos de irrigação. Brasília: Programa Nacional de Irrigação, 1986.p.T11.
Olitta, A.F.L. Os métodos de irrigação. São Paulo: Livraria Nobel, 1987.287p.

Pizarro, F. Riegos localizados de alta frecuencia: Goteo, microaspersion, exudacion. 2. ed. Madrid: Ediciones Mundi-Prensa, 1990. 461p.

SAEG- Sistema para análise estatística. V.5-0. Viçosa: Fundação Arthur Bernardes, UFV, 1993.

Sampaio, S.C.; Vilela, L.A.A.; Dias, J.A.A.; Faria, M.A. de. Características hidráulicas e da distribuição de água do microaspersor NAAN 7110. Ciência e Agrotecnologia, Lavras, v.20, n.1, p.70-78, 1996.

Solomon, K. Manufacturing variation of trickle emitters. Transactions of the ASAE, St. Joseph, v.22, n.5, p.1034-1038, 1979.

Telles, D.D. Projeto de irrigação localizada. In: Curso de elaboração de projetos de irrigação. Brasília: Programa Nacional de Irrigação. 1986. p.T11. 